\title{
Die organischen Substanzen der Schaalen von Mytilus und Pinna.
}

\author{
Von
}

G. Wetzel,

Assistent am physiologischen Institut.

(Aus dem physiologischen Institut zu Breslau.)

(Der Redaction zugegangen am 7. April 1900.)

Unter Systematik sind wir gewohnt, eine Anordnung der Geschöpfe nach den Eigenthümlichkeiten ihrer Gestalt zu verstehen. Ich bezweifle nicht, dass für praktische Zwecke dies das allein mögliche Princip ist. Aber wir könnten uns auch eine Klassifikation auf Grund des Vorkommens bestimmter Stoffe oder des Auftretens bestimmter Arten stofflicher Umwandlungen denken. In der Systematik der Spaltpilze spielen derartige Principien eine nicht unbedeutende Rolle. So das Verhalten der Bakterien $\mathrm{zu}$ verschiedenen Farbstoffen, ihr Verhalten zu dem Nährboden, und die Anforderungen, die sie an denselben stellen. ${ }^{1}$ ) Bei der systematischen Verwerthung von Differenzen in chemischen Vorgängen ist indessen die grösste Vorsicht geboten. Viele scheinbar charakteristische Erscheinungen sind nur von äusseren Bedingungen abhängig und wechseln mit ihnen. So schien die grössere Widerstandsfähigkeit des Hundes gegen die Folgen von Säurezufuhr gegenüber der des Kaninchens in der Organisation des Hundes begründet zu sein. Sie war aber nur eine Folge der Fleischnahrung und der mit Pflanzennahrung gefütterte Hund verhält sich wie das Kaninchen. Ebenso wechselt die Pigmentproduktion von Bakterien mit ihren äusseren Existenzbedingungen.

1) Flügg e, Mikroorganismen. Bd. II. 
Hoppe-Seyler ${ }^{1}$ hat für den Satz, dass die Ontogenie gleich der Phylogenie ist, auf Bestätigungen in der Chemie der Organismen hingewiesen. Er sagt: "Geht man die $\mathrm{Zu}$ sammensetzung der Gewebe vergleichend von den niedriger organisirten $\mathrm{zu}$ den höher entwickelten Thieren fortschreitend durch, so findet man zuerst das Auftreten von mucingebendem Gewebe, dann bald chondringebendem, endlich auch in den Cephalopoden das Auftreten von glutingebendem Gewebe.» «Ganz dieselbe Reihenfolge ergibt sich, wenn man die Stadien der Entwicklung eines Embryo, z. B. des Hühnchens im Ei, verfolgt, und ich kann mir nicht denken, dass diese Uebereinstimmung nur eine zufällige sei.» - Ebenso weist HoppeSeyler darauf hin, dass die morphologische Klassifikation der Organismen durch eine Berücksichtigung ihrer chemischen Eigenthümlichkeiten eine Ergänzung erfahren könne.

Die hiermit in grossen Zügen angedeuteten Gebiete zur Verwerthung der Resultate der physiologisch-chemischen Forschung bedürfen zu ihrer näheren Kenntniss noch einer eingehenden Untersuchung aller in den verschiedenen Klassen des Thierreichs vorkommenden gewebsbildenden Substanzen. Die endgültige Entscheidung darüber, ob aus den chemischen Eigenthümlichkeiten der Geschöpfe sich Gesetzmässigkeiten ableiten lassen, welche mit den auf morphologischem Gebiete gefundenen parallel gehen, wird sich erst dann sicher treffen lassen.

In dem hier angedeuteten Sinne möchte ich die in Folgendem mitgetheilten Untersuchungen über das Conchiolin in erster Linie aufgefasst sehen.

Ich musste meinen Untersuchungen aus äusseren Gründen einen Abschluss geben. Daher sah ich mich gezwungen, manche Theile, vor Allem die Erörterung über die Stellung des Conchiolins unter den Albuminoïden und im Zusammenhange damit die Frage nach seinem Schwefelgehalt, auf dem Punkte stehen zu lassen, auf dem sie sich gerade befanden, ohne sie zu einem sicheren definitiven Ende führen zu können.

1) Pflüg er's Archiv. Bd. 14. 1877. S. 400. 
Unsere gegenwärtigen Kenntnisse über das Conchiolin beruhen auf den älteren Untersuchungen von Fremy, Kost, Schmidt, Schlossberger und C. Voit, sowie auf den neueren von Krukenberg und Engel.

Fremy hat (nach Schlossberger) die Bezeichnung Conchiolin für die organische Gerüstsubstanz der Lamellibranchiaten eingeführt. Später stellte zunächst Schlossberger ${ }^{1}$ ) fest, dass das Conchiolin mit Chitin, wofür es damals gehalten wurde, nichts zu thun habe. Der Stickstoffgehalt des Chitins beträgt nach ihm 6,5\%, der des Conchiolins über $16 \%$.

Einen Fortschritt in der Erkenntniss der Stellung des Conchiolins bildete ferner die Untersuchung C. Voit's ${ }^{2}$ ) welcher unter anderen Thatsachen feststellte, dass das Conchiolin aus den Schaalen der Perlmuschel sich mit Millon's Reagens roth färbte. Voit behandelte den nach der Entkalkung der Schaalen erhaltenen Rest mit verdünnter Kalilauge in der Kälte und stellte mit dem darin unlöslichen Antheil die genannte Reaction an. ${ }^{3}$ )

Hiermit stellten sich später die Untersuchungen Krukenberg's ${ }^{4}$ ) in Widerspruch. Dieser Forscher gab an, dass das Conchiolin keine Rothfärbung mit Millon'schem Reagens gäbe, und dass sich unter seinen Zersetzungsprodukten kein Tyrosin befände. Diese Angaben erklären sich vielleicht dadurch, dass Krukenberg als Conchiolin einen Stoff untersucht hat, der die Eihüllen der Murexeier bildet. Er nennt diesen Stoff ein sehr reines Conchiolin. Die Eihüllen behandelte er mit Salzberg 1856.

1) Schlossberger, Chemie der Gewebe. Leipzig u. Heidel-

2) Zeitschr. f. wissenschaftl. Zoologie. Bd. X. 1860.

3) Bei Voit und bei Schlossberger findet sich auch die übrige ältere Litteratur citirt.

4) Zeitschr. f. Biologie. Bd. 22. Berichte d. d. ch. G. Bd. XVIII. Vergl. Physiologische Vorträge. Heidelberg 1885.

Anmerkung. Wie ich nachträglich sehe, hat Simroth neuerdings den Vorschlag gemacht, die Bezeichnung Conchiolin durch «Conchin zu ersetzen. Zoolog. Anz. Bd. 20. S. 471. 
säure, unterwarf sie der Pepsin- und der Trypsinverdauung und der Maceration in verdünnter Natronlauge. Diese Vorbehandlung entspricht also mit Ausnahme der Verdauungen dem Verfahren Voit's. Krukenberg war ausserdem der erste, welcher die Zersetzungsprodukte seines Conchiolins studirte. Er gibt an, nur Leucin und Leucinimid nebst einem in klaren Prismen krystallisirenden unbekannten Körper gefunden $\mathrm{zu}$ haben. Das Auftreten von Tyrosin und Glykokoll stellt er in Abrede.

Die Eischaalen von Murex wurden später noch einmal von Engel ${ }^{1}$ ) untersucht. Er fand, dass sie nach der Behandlung mit den verschiedensten Reagentien stets noch Rothfärbung mit Millon'schem Reagens gaben. Er kam schliesslich zu dem Resultat, dass die Murexeischaalen einen schwefelhaltigen keratinartigen und ausserdem einen schwefelfreien Stoff enthalten. Von dem letzten gibt er die Möglichkeit zu, dass er mit dem Conchiolin Krukenberg's identisch sein könne. Er gab aber ebenfalls noch Rothfärbung mit Millon's Reagens. Schliesslich bestätigt Fingel noch die Angaben Voit's, dass auch das Conchiolin in der Perlmuschel diese Reaction gäbe. Die Zersetzungsprodukte des Conchiolins hat Engel nicht untersucht.

Die Lehrbücher der physiologischen Chemie von $\mathrm{Ham-}$ marsten und von Neumeister, sowie das Handbuch von Hoppe-Seyler geben an, dass das Conchiolin keine Rothfärbung mit Millon'schem Reagens gäbe. Auch in Gautier's "Leçons de chimie biologique ${ }^{2}$ ) wird das Eintreten der Rothfärbung verneint. Ebenso fehlt nach Angabe dieser Werke das Tyrosin unter den Zersetzungsprodukten. Nach Gautier soll Glykokoll sich darunter befinden, während die übrigen Lehrbücher nur Leucin angeben. Ich weiss nicht, auf welche Litteraturangabe diese Angabe Gautier's zurückgeht oder ob sie auf eigener Untersuchung beruht. Gautier bemerkt ausserdem, dass er etwas Schwefel im Conchiolin gefunden habe.

1) Zeitschr. f. Biologie. Bd. 27, S. 374 u. Bd. 28, S. 345.

2) Paris 1897. 
Das Material für meine eigenen Untersuchungen habe ich auf der zoologischen Station zu Neapel gesammelt und die Arbeit in dem chemischen Laboratorium der Anstalt begonnen. Für die reichliche Versorgung mit dem hier verarbeiteten wie mit vielem andern Material bin ich Herrn Dr. Lo Bianco zu grossem Danke verpflichtet. Ebenso gebührt mein Dank dem damaligen Leiter der physiologischen Abtheilung, dem verstorbenen Physiologen K. Schönlein. Es entspricht seinem ausdrücklichen Wunsche, wenn ich hier darauf hinweise, dass der physiologische Chemiker in der Station in der Villa nazionale ein gut ausgerüstetes chemisches Laboratorium vorfindet, welches ihm die vollständige Ausführung chemischer Arbeiten gestattet. Der Aufenthalt in Neapel wurde mir durch ein Stipendium aus der Gräfin Luise Bose-Stiftung ermöglicht, Der preussischen Regierung bin ich wegen der Ueberlassung eines Arbeitsplatzes an der Station zum grössten Danke verpflichtet.

Als Object für meine Untersuchungen habe ich hauptsächlich die Schaalen von der Miesmuschel, Mytilus edulis, und zwar der im Mittelmeer lebenden Varietät galloprovincialis und die Schalen der riesigen rothen Steckmuschel, Pinna nobilis, verwendet. Daneben dienten die Eischaalen verschiedener Schnecken zur Untersuchung. Jedoch wurde durch diese die Arbeit nur wenig gefördert, da es nicht möglich war, sie in so grosser Menge zu beschaffen wie die Schaalen der Muscheln. Dies musste aber für die Untersuchung der Zersetzungsprodukte, an der mir in erster Linie gelegen war, zur Vorbedingung gemacht werden.

Die Mytilusschaalen bestehen aus mehreren, durch ihre Struktur unterschiedenen Schichten, welche einen organischen Rest von verschiedenem Aussehen liefern, wenn man sie mit Salzsäure entkalkt. Ist es nun richtiger, den gesammten organischen Rest ohne Sonderung der einzelnen Bestandtheile $\mathrm{zu}$ untersuchen oder das Conchiolin jeder morphologisch einheitlichen Substanz für sich zu sammeln? Ich habe mich, was die Untersuchung der Spaltungsprodukte anbelangt, für die Untersuchung des Gesammtrestes entschieden und zwar deswegen, 
weil hierzu eine grössere Menge Substanz unerlässlich ist, deren Beschaffung ausserordentliche Schwierigkeiten machen würde, wenn man die einzelnen Bestandtheile der Schaale sondern und für sich sammeln wollte. Ferner ist die Perlmuttersubstanz, für welche sich am ehesten die Möglichkeit einer Verschiedenheit von der übrigen Schaale eröffnet, verhältnissmässig nur in sehr geringer Menge vorhanden. Bei der Untersuchung der Spaltungsprodukte sind also diese, soweit sie in grösserer Menge sich finden, auf die Hauptmasse der Schaalensubstanz $\mathrm{zu}$ beziehen.

Ausserdem aber habe ich von einer kleinern Menge von Schaalen die einzelnen Substanzen gesondert, um sie für sich einer orientirenden Untersuchung zu unterziehen. Die Resultate dieser Untersuchung will ich der der Spaltungsprodukte voraufschicken.

Aus den Schaalen von Pinna erhält man zwei verschiedene Produkte. Die Grundsubstanz ihres roth gefärbten Hauptantheiles bildet nach der Entkalkung ziemlich feste, zusammenhängende Häute oder Lappen, welche ein Fachwerk darstellen. Die Hohlräume der Häute enthielten vorher die Hauptmasse des kohlensauren Kalkes. Ausser diesem Produkt gewinnt man ein weisses, sehr viel feineres und nicht so zusammenhängendes aus der Perlmuttersubstanz. Diese weissen Flocken werden in viel geringerer Menge erhalten. Die Analysen beider Schaalen ergaben gewisse Unterschiede.

Die zur Analyse verwendeten rothen Häute wurden nach der Entkalkung noch 24 Stunden mit einer etwa 1\% von Natronhydrat in der Kälte behandelt und nacheinander der Verdauung mit Pepsinsalzsäure und mit Pankreassaft unterworfen. Bei diesen Proceduren ging eine geringe Substanzmenge in Lösung, welche nicht quantitativ bestimmt worden ist. Die so vorbehandelte Substanz wurde dann mit Alkohol und mit Aether extrahirt und getrocknet. Die Perlmuttersubstanz wurde nach der Entkalkung und nachdem sie kalkfrei gewaschen war, sogleich mit Alkohol und Aether behandelt. Die Unterschiede in der Behandlung beider Substanzen dürften kaum hinreichen, um die Verschiedenheiten in 
der Zusammensetzung zu erklären, welche sich in den folgenden Analysenresultaten zeigen.

\begin{tabular}{|c|c|c|c|}
\hline $\begin{array}{c}\text { Bei } 1250-1300 \\
\text { getrocknet und } \\
\text { aschefrei berechnet }\end{array}$ & $\begin{array}{l}\text { Rothes Conchiolin } \\
\text { Weise Perlmutter- } \\
\text { grundsubstanz }\end{array}$ & $52,87 \% \mathrm{C}$ & $6,54 \% \mathrm{H}$ \\
\hline
\end{tabular}

Im Stickstoffgehalt fand sich kein Unterschied. Der hauptsächlichste Unterschied liegt im Kohlenstoffgehalt. Beide Substanzen enthielten ausserdem Schwefel. Sie gaben bei der Schön'schen Schwefelprobe eine Violettfärbung mit Nitroprussidnatrium. Der Schwefel im rothen Conchiolin wurde auch quantitativ bestimmt. Die rothen Schaalen hinterliessen auf dem Platinbleche einen beträchtlichen Ascherückstand, welcher bestimmt und zu 1,76\% gefunden wurde. Die weisse Substariz enthielt nur schr geringe Spuren von Asche, welche, auf das Analysenresultat von keinem merklichen Einfluss sein konnten. Als Resultat ergibt sich also, dass das Perlmutterconchiolin einen geringeren Kohlenstoffgehalt besitzt als das rothe Conchiolin.

Die Mytilusschaalen geben drei verschiedene Häute. Wird eine Schaale von Mytilus vollständig entkalkt, so stellt sie ein schlaffes Gebilde dar, welches die Form der harten Schaale noch leidlich beibehalten hat. In der spitzen Hälfte befindet sich an der Innenseite ein Häufchen weisser, lockerer Masse. Diese stammt aus der Perlmuttersubstanz der Schaale. Das Uebrige besteht aus zwei gleich grossen Häuten, einer äusseren und einer inneren, welche sich leicht von einander abziehen lassen. Die innere ist gleichmässig dunkelbraun gefärbt. Auf ihrer Oberfläche sitzen noch Theile der weissen Substanz, welche daran so fest haften, dass sie sich nicht wohl entfernen lassen. Die äussere Haut zeigt concentrische, abwechselnd tief dunkel und etwas heller gefärbte Ringe. An den helleren Stellen ist sie mit bräunlich-gräulicher Farbe durchscheinend. Sie ist sehr fest gegenüber der inneren Haut. Von den drei Häuten lassen sich mithin als einheitlich nur die äussere dunkle und die Perlmutiersubstanz ansehen. Von der Untersuchung der innern dunklen Haut, welche noch mit Theilen der Perlmuttersubstanz verbunden ist, habe ich aus diesem Grunde 
ganz abgesehen. Von der weissen Substanz erhielt ich aus $100 \mathrm{~g}$ Schaalen nur annähernd 1 Decigramm. Mit dieser Menge habe ich keine Analyse gemacht. Die schwarzen äusseren Häute wurden mit Wasser gewaschen, bis das Filtrat mit oxalsaurem Ammoniak keine Trübung mehr gab, dann mit Alkohol und mit Aether extrahirt, getrocknet und verrieben. Der Kohlenstoffgehalt dieser Substanz war ebenfalls beträchtlich höher als der der Perlmuttersubstanz von Pinna. Ein direkter Vergleich mit der Perlmuttersubstanz von Mytilus konnte nicht gemacht werden.

In den von Schlossberger und Voit untersuchten Schaalen der Auster und der Perlmuschel finden sich ebenfalls Häute von verschiedener Beschaffenheit.

Die Schaalen der Auster liefern nach Schlossberger ${ }^{1}$ ) zwei Arten von Substanzen, erstens «braune, derbe, etwas durchscheinende Häute» und zweitens "weisse oder weissgraue Flocken». Die letzteren stammen hier ebenfalls zum grossen Theil aus der Perlmuttersubstanz. Schlossbergers braune Häute würden der äussern und innern Haut zusammen entsprechen. Ueber die chemische Natur der beiden Häute nimmt er Folgendes an. In den braunen sind zwei Substanzen enthalten, eine in Kalilauge lösliche und eine darin völlig unlösliche Substanz. Die weissen Häute hält er für einen chemisch einheitlichen Körper, der dem löslichen Antheil der braunen Häute sehr nahe stehe. Die leichtere Löslichkeit der Perlmuttersubstanz in Alkalien und Säuren kann ich für Mytilus und Pinna bestätigen.

Die Perlmuschel liefert nach G. Voit ${ }^{2}$ ) ebenfalls ein braunes und ein weisses Conchiolin, von denen sich das braune schwerer in Alkalien löst als die weissen Häute, er kommt jedoch $\mathrm{zu}$ dem Schluss, dass sie sich in ihrem chemischen Verhalten nicht wesentlich von einander unterscheiden.

1) Chemie d. Gewebe s. o.

2) Ztschrft. f. wissenschaftl. Zoologie Bd. X. 
Zum Studium der Zersetzung sprodukte des Conchiolins mit Säuren habe ich ausschliesslich die Schaalen von Mytilus verwendet. Wie oben schon auseinandergesetzt, wurde $\mathrm{zu}$ diesem Zwecke keine Trennung der einzelnen Arten von Häuten vorgenommen, sondern der gesammte organische Rest verwendet. Im Ganzen habe ich, um über grössere Substanzmengen verfügen zu können, etwa einen Centner Miesmuschelschaalen entkalkt.

Die Schaalen sind zum Theil mit den aus dem Byssusorgan stammenden gelben, sehr zähen Byssusfäden besetzt. Viele tragen auch die Gehäuse von Röhrenwürmern oder die Stöcke kleiner Hydroidpolypen oder die Gehäuse von Seepocken. Solche Schaalen wurden ausgesondert und fortgeworfen. Die mit viel Byssus besetzten wurden für sich entkalkt, ebenso die davon mehr oder weniger freien. Wo der Byssus in einem dichten Knäuel aufsass, wurde er abgerissen und besonders gesammelt. Zur Entkalkung diente eine verdünnte Salzsäure, welche im Liter zwischen 5 und $10 \mathrm{ccm}$. concentrirter Salzsäure enthielt. Die. Säure befand sich in grossen grünen Glasballons. Aus diesen wurde die verbrauchte Säure abgehebert und durch frische ersetzt, bis die Schaalen nahezu vollständig entkalkt waren. Die gänzliche Entkalkung konnte an den nunmehr an Volumen sehr zusammengeschwundenen Massen in grösseren Glascylindern und Bechergläsern ausgeführt werden. Die vollständig entkalkten Schaalen wurden in Leinenbeuteln ausgepresst, wiederholt in Leitungswasser suspendirt und wieder ausgepresst. Dies geschah so lange, als die Flüssigkeit noch sauer reagirte und ihr Kalkgehalt den des verwendeten Leitungswassers übertraf. Dann wurden die Schaalen in derselben Weise mit destillirtem Wasser ausgewaschen, bis die Flüssigkeit mit Ammoniak und Ammoniumoxalat keine Fällung mehr gab.

An Stelle des Auspressens durch einen Beutel bediente ich mich in den späteren Stadien der Reinigung des Absaugens auf der Nutsche. Die Schaalen sind nämlich zuletzt zum Theil schon in sehr feine Bruchtheile zerfallen, von welchen beim öfteren Auspressen durch Leinwand nicht unbeträchtliche Mengen 
verloren gehen würden. Die feinsten Bruchstücke wurden auch zum Theil für sich gewonnen. Sie senken sich zu Boden und die grösseren können zunächst mit der Hauptmasse der Waschflüssigkeit auf das Filter gebracht werden. Die feinen Theile lässt man dann sedimentiren, wäscht durch Dekantiren aus und saugt sie schliesslich auch auf dem Filter ab. Grobe und feine Theile wurden natürlich schliesslich wieder vereinigt.

Die so gewonnene Masse bestand jetzt aus verschieden gefärbten Häuten, die noch eine ziemlich elastische Beschaffenheit zeigten und sich nur sehr unvollkommen in der Reibschaale zerkleinern liessen.

Die Häute wurden nun zweimal mit Alkohol und einmal mit Aether je 24 Stunden stehen gelassen. Der Aether wurde abgesaugt und abgepresst und die Massen auf dem Wasserbade getrocknet. Jetzt erhielt ich eine ziemlich spröde Substanz. Sie liess sich bequem zu einem schwarzbraunen Pulver verreiben. Das Pulver stäubt leicht und besteht aus ganz feinen Theilchen und etwas grösseren Blättchen. Dazwischen befinden sich noch gelbliche Blättchen, die Anheftepünktchen der Byssusfäden. Ferner finden sich nun noch, besonders in der einen Hälfte der Präparate, die Byssusfäden selbst und einige grössere Verunreinigungen. Diese und die Byssusfäden lassen sich durch Sieben vermittelst eines feinen Siebes abtrennen.

Ich erhielt also im Ganzen zwei Präparate. Das eine, reine, enthielt neben den Schaalen nur wenig Byssus, das andere eine etwas grössere Menge dieser Substanz. Ausserdem enthielten sie noch hornige Ränder vom Scharnier der Schaalen. Die gesammte Ausbeute betrug etwas mehr als ein halbes Kilo, also etwa $1 \%$ der verwendeten Schaalenmasse.

Bisher habe ich nur $100 \mathrm{~g}$ von dem reineren Theil der Ausbeute zersetzt. Das übrige Material ist nur zum kleinen Theil für andere Bestimmungen und Untersuchungen verarbeitet. Von dem reineren Präparat habe ich eine vollständige Analyse gemacht. Sie ergab folgende Zahlen (für bei $125--130^{\circ}$ getrocknete Substanz):

$\begin{array}{cccc}\mathrm{C} & \mathrm{H} & \mathrm{N} & \mathrm{S} \\ 52,3 \% & 7,6 \% & 16,4 \% & 0,65 \%\end{array}$


Die $100 \mathrm{~g}$ wurden 24 Stunden lang mit einer Schwefelsäure, welche auf 1 Theil Säure 2 Theile Wasser enthielt, am Rückflusskühler gekocht. Ein schwarzer pulveriger Bodensatz aus organischer Substanz, welcher nicht in Lösung gegangen war, wurde abfiltrirt. Auf die Untersuchung dieser Substanz werde ich unten zurückkommen. Darauf wurde die Flüssigkeit verdünnt und mit Phosphorwolframsäure zur Entfernung und Gewinnung der basischen Spaltungsprodukte gefällt.

Den Phosphorwolframsäure-Niederschlag habe ich mit Barythydrat zerlegt und aus dem Filtrat das Gemisch der Basen gewonnen, jedoch habe ich diesen Theil der Zersetzungsprodukte qualitativ nicht bearbeitet. Hingegen habe ich schon früher eine Bestimmung der Menge des durch Phosphorwolframsäure fällbaren Stickstoffs vorgenommen und publicirt. $\left.{ }^{1}\right)$

Das Filtrat vom Phosphorwolframsäure-Niederschlag wurde, mit Barythydrat ausgefällt und sowohl die Phosphorwolframsäure als die Schwefelsäure entfernt. Den Ueberschuss von gelöstem Baryt entfernte ich aus der von den Baryumniederschlägen abfiltrirten Flüssigkeit durch genaues Neutralisiren mit Schwefelsäure.

Das eingedampfte Filtrat vom schwefelsauren Baryt schied beim Erkalten grosse Mengen weisser Krystallmassen aus, welche unter dem Mikroskop lange, zum Theil sternförmig zusammengelegte, zum Theil büschelig angeordnete Nadeln zeigten. Die heisse Lösung des Stoffes nahm Kupferoxydhydrat zu einer blauen Lösung auf, die sich beim Sieden trübte und eine schwarze Ausscheidung lieferte. Die Krystalle gaben intensive Rothfärbung mit Millon'schem Reagens. Es war zu erwarten, dass Tyrosin vorlag. Die Substanz enthielt zunächst noch ein wenig Asche, welche sich durch Umkrystallisiren aus heissem Wasser bis auf sehr geringe Spuren entfernen liess. Von dem so gereinigten Präparat wurde eine Stickstoffbestimmung gemacht.

$0,1536 \mathrm{~g}$ Substanz brauchten zur Neutralisation $3,61 \mathrm{ccm}$. einer $1 / 4$ Normalnatronlauge, von der $1 \mathrm{ccm} 3,2115 \mathrm{mg} \mathrm{N}$ entsprach.

$\begin{array}{ccc} & \% \mathrm{~N} \text { berechnet } & \% \mathrm{~N} \text { gefunden } \\ \text { Tyrosin } & 7,75 & 7,55\end{array}$

1) Diese Zeitschrift, Bd. XXVI. 
Danach dürfte das Vorhandensein von Tyrosin, welches Krukenberg in seinen Substanzen nicht gefunden hatte und welches man in Folge des Auftretens der Millon'schen Reaction unbedingt erwarten musste, erwiesen sein.

Die Menge des aus $100 \mathrm{~g}$ Substanz gewonnenen unreinen Tyrosins betrug etwa $5 \mathrm{~g}$.

Die nach dem Auskrystallisiren des Tyrosins übrig bleibenden Amidosäuren habe ich im Wesentlichen nach dem Verfahren von Schulze ${ }^{1}$ ) behandelt. Ich bin von ihm insofern abgewichen, als ich statt des Kupferoxydhydrates Kupfercarbonat verwendel habe. Ich erhielt beim Kochen der Lösungen der Amidosäuren mit Kupfercarbonat stets klare blaue Lösungen, aus denen erst beim Erkalten mehr oder weniger auskrystallisirte. Hierdurch unterschieden sich die aus dem Conchiolin gewonnenen Amidosäuren sehr erheblich von Amidosäurenfractionen, welche gleichzeitig im Laboratorium aus Casein dargestellt worden waren und mit Kupferoxydhydrat erhitzt wurden. Es bildete sich hier schon in der heissen Lösung ein voluminöser Niederschlag.

Zunächst wurden aus den nach Entfernung des Tyrosins übrig bleibenden Amidosäuren so viel Krystallfractionen gewonnen, als daraus in der Kälte nach allmählich stärkerem fortgesetzten Eindampfen auskrystallisiren wollten. Es blieb schliesslich ein dicker Syrup übrig, welcher noch sehr viel Substanz enthielt. Diesen habe ich noch nicht genügend untersuchen können. Die durch Auskrystallisiren gewonnenen Fractionen wurden nun einzeln in ihre Kupfersalze verwandelt und diese fractionirt. Dabei wurden stets die Substanzen, welche etwa eine gleiche Löslichkeit zeigten, aus verschiedenen Fractionen mit einander vereinigt, um die Anzahl der Fractionen möglichst wenig zu erhöhen.

Schliesslich erhielt ich einerseits einige Fractionen, es waren die späteren, welche hauptsächlich nur ein einziges, in heissem Wasser leicht lösliches Kupfersalz enthielten. Es krystallisirte beim Erkalten in langen Nadeln aus. Die Krystalle zeigten mikroskopisch eine sehr gleichmässige Beschaffenheit.

1) Diese Zeitschrift, Bd. IX, S. 63 u. 253. 
Die ersten Fractionen dagegen zeigten hier und da auch nadelförmige Krystalle, in der Hauptsache aber keine ausgesprochene Krystallform. Hier war also noch ein Gemisch vorhanden. Ich reinigte es jetzt nicht mehr durch Umkrystallisiren, sondern dadurch, dass ich die einzelnen Krystallfractionen abfiltrirte und mit wenig Wasser auskochte. Das Lösliche wurde stets vereinigt und ebenso das Unlösliche. Aus den gelösten Antheilen wurden durch Auskrystallisiren noch wieder unlösliche gewonnen und das ganze Verfahren noch einigemale wiederholt. So erhielt ich ziemlich rasch ein fast ganz unlösliches Kupfersalz. Es wurde mit Schwefelwasserstoff zersetzt, wobei sich das Schwefelkupfer erst nach wiederholtem Eindampfen und neuem Zusatz von Wasser vollständig ausschied. Aus der abfiltrirten eingedampften Flüssigkeit krystallisirten grosse vielzackige Blättchen, welche in Drusen angeordnet waren. Die Krystalle waren in heissem Alkohol löslich und krystallisirten daraus beim Erkalten in schönen sechsseitigen Täfelchen, der Krystallform des reinen Leucins. Der Körper sublimirte beim Erhitzen im Reagensglase ohne Rest. Im Schmelzröhrchen verschwanden sie bei langsamem Erhitzen ebenfalls allmählich bis auf einen geringen braunen Rückstand, bei schnellem Erhitzen trat bei $170-171^{\circ}$ Zersetzung und dann ebenfalls rasches Verschwinden ein. Der Stickstoffgehalt

- des Präparates wurde nach Kjeldahl bestimmt.

$0,2142 \mathrm{~g}$ Substanz entsprachen $7,92 \mathrm{ccm}$. einer $1 / 4$ Normallauge, von der $1 \mathrm{ccm} .3,2115 \mathrm{mg} \mathrm{N}$ entsprach.

$\begin{array}{ccc}\text { Leucin (Amidocapronsäure) } & \% \mathrm{~N} \text { berechnet } & \% \mathrm{~N} \text { gefunden } \\ 10,69 & 10,6\end{array}$

Das Leucin ist schon von Krukenberg unter den Zersetzungsprodukten des Conchiolins aufgefunden, jedoch nicht hinreichend sicher gekennzeichnet worden.

Die erhaltene Menge betrug rein nur einige Decigramm, auch die des unreinen Leucins war nur gering.

Wir wenden uns nun zu dem Kupfersalz der letzten Fractionen, welches die erwähnten langen Nadeln bildete. Das Kupfersalz. wurde mit Schwefelwasserstoff zersetzt. Hierbei schied sich das Schwefelkupfer im Gegensatz zu den ersten 
Leucinfractionen sofort vollständig aus und setzte sich gut unter der wasserhellen Flüssigkeit ab. Das eingedampite Filtrat schied starke Prismen aus, welche zum Theil in Drusen angeordnet waren. Es konnten also Glykokollkrystalle sein.

Nach einmaligem Umkrystallisiren zeigte die Substanz folgende Eigenschaften. Sie bräunte sich beim Erhitzen von $225^{\circ}$ an stark und begann sich unter Gasentwicklung bei 234 bis $235^{\circ} \mathrm{zu}$ zersetzen. Nach Hoppe-Seyler soll das Glykokoll sich bei $228^{\circ}$ bräunen und bei $232-236^{\circ}$ zersetzen. Ich habe dann. noch das Thiohydantoïn des vermuthlichen Glykokolls dargestellt. $0,5 \mathrm{~g}$ des Körpers wurden mit 0,37 $\mathrm{g}$ Kalihydrat in Alkohol verrieben und zu einer alkoholischen Lösung von $0,9 \mathrm{~g}$ Phenylsenföl gesetzt. Beim Erwärmen auf dem Wasserbade entstand ein Brei, der abfiltrirt, in Wasser gelöst und mit HCl übersättigt wurde. Beim Erhitzen der Lösung schied sich das Thiohydantoïn aus. Es wurde abfiltrirt und sein Schmelzpunkt bestimmt. Es schwärzte sich von $200^{\circ}$ ab zunehmend, ohne zu schmelzen und ohne Gasentwicklung, jedoch unter Bildung brauner Destillationsprodukte. Dies stimmt mit den Angaben Aschan's') überein.

Schliesslich habe ich noch eine Stickstoffanalyse der freien Amidosäure und eine Krystallwasserbestimmung des Kupfersalzes ausgeführt. Die Bestimmungen ergaben

$\begin{array}{ccc}\text { Glykokoll } & \% \mathrm{~N} \text { berechnet } & \% \mathrm{~N} \text { gefunden } \\ & 18,67 & 18,6 \\ \text { Krystallwasser } & \% \text { Krystallwasser } \\ & \% \text { berechnet } & \text { gefunden } \\ & 7.85 & 7,69\end{array}$

Mit diesen Resultaten ist das Vorhandensein des Glykokolls sicher gestellt.

Wie schon oben erwähnt, gibt auch Gautier in seinem Lehrbuch an, dass das Conchiolin Glykokoll liefere. Seine Quelle für diese Angabe ist mir unbekannt geblieben.

Die Menge des gefundenen Glykokolls war erheblich und wird schätzungsweise etwa $4 \mathrm{~g}$ betragen haben.

1) B. d. d. ch. G. Bd. XVII. 
Von weiteren Körpern ist in den letzten Fractionen in geringer Menge ein reducirender Körper vorhanden, welcher sich durch die Bildung von wenig rothem Kupferoxydul beim Eindampfen der Kupfersalzlösungen bemerklich macht. Ich habe nicht versucht, den Körper zu isoliren. Da weder Leucin noch Glykokoll gelöstes Kupferoxydhydrat beim Kochen reduciren, so muss es ein von ihnen verschiedener Körper sein.

Besondere Aufmerksamkeit habe ich noch dem Nachweise der von Schulze entdeckten Phenylamidopropionsäure ${ }^{1}$ ) gewidmet. Den Stoff selbst habe ich, wie aus dem Obigen ersichtlich ist, unter den Leucinfractionen nicht auffinden können. Ich habe daher untersucht, ob sich vielleicht bei der Oxydation einer Fraction des Rohleucins Benzoësäure bildet. Da Schulze angibt, dass die Phenylamidopropionsäure sich in der Regel in den späteren Fractionen findet, so habe ich eine von diesen, Fraction IV, deren Menge $1 \mathrm{~g}$ betrug, mit Kaliumbichromat und verdünnter Schwefelsäure gekocht. Beim Erkalten schied sich nichts aus. Die Reactionsflüssigkeit wurde nun mit Aether extrahirt. Als Verdunstungsrückstand hinterblieben wenige Tropfen, welche einen fettsäureartigen, üblen Geruch besassen. Eine als Benzoësäure anzusprechende Substanz war in diesem Versuche also nicht aufzufinden. Um jedoch auch ganz geringe Mengen einer Phenylamidosäure nicht zu übersehen, wurden in einem besonderen Versuche $10 \mathrm{~g}$ Mytilus-Conchiolin von dem reinen Präparate mit Schwefelsäure zersetzt, der schwarze Bodensatz abfiltrirt und nach Entfernung der Schwefelsäure mit Baryt auch das Tyrosin auskrystallisiren gelassen. Das gesammte übrig bleibende Amidosäurengemisch wurde mit Schwefelsäure und Kaliumbichromat oxydirt. Die Reactionsflüssigkeit roch deutlich nach Benzoë, schied aber beim Erkalten keine Benzoësäure aus. Durch Ausschütteln mit Aether und Verdunstenlassen des Aethers liess sich ein Rückstand von wenigen glänzenden, gezackten Blättchen gewinnen, welche somit wahrscheinlich Benzoësäure

1) Diese Zeitschrift, Bd. IX, S. 63. 
waren. Es muss nach diesem Resultat angenommen werden, dass das untersuchte Rohconchiolin geringe Mengen einer Phenylamidosäure liefert. Jedoch sind diese Mengen wahrscheinlich so gering, dass man sie nur auf die dem Conchiolin in kleinen Mengen beigemengten andern Stoffe beziehen kann. - Parallel mit den Oxydationen der Leucinfractionen habe ich solche mit Mandelsäure ausgeführt und bin schon bei Verwendung weniger Decigramme zu Benzoëmengen gelangt, die zum Nachweis der Identität durch den Schmelzpunkt ausreichten.

Das Rohconchiolin, welches zum Studium der Zersetzungsprodukte verwendet worden ist, enthielt $0,65 \%$ Schwefel. Der Schwefel braucht nicht auf das Conchiolin selbst bezogen zu werden, sondern kann sehr wohl aus den Beimengungen herstammen, obwohl seine Menge nicht ganz gering ist.

Das reine Conchiolin aus Murexeischaalen soll nach Krukenberg keinen Schwefel enthalten. Ebensowenig enthält ihn das von V oit untersuchte Conchiolin aus den Schaalen der Perlmuschel. Dies letztere hat Engel noch einmal bestätigt. Die Häute der Perlmuschel lösen sich nach ihm beim Kochen in Kalilauge auf. Diese alkalische Lösung enthält keinen Schwefel. - Die Grundsubstanz der Murexeischaalen, welche nach Krukenberg reines Conchiolin sein soll, besteht nach Engel zum Theil aus einem schwefelhaltigen Keratin, zum Theil aus einem in Alkalilauge schwerer löslichen, schwefelfreien Stoff. Gautier fand im Conchiolin etwas Schwefel. (Siehe oben S. 389.)

Unter den mir vorliegenden Substanzen sehe ich das Conchiolin aus den rothen Pinnaschaalen als das reinste an. Das aus den Mytilusschaalen enthält eine beträchtliche Menge des schwarzen Pigmentes, welches sich ohne Zerstörung der Schaalen nicht extrahiren lässt. Der rothe Farbstoff dagegen, welchen die Pinnaschaalen enthalten, lässt sich grösstentheils mit Alkohol extrahiren. Vollständig lässt auch er sich nicht entfernen, da die Schaalen stets einen röthlichen Ton behalten. Das zur Analyse verwendete Conchiolin war kalkfrei gewaschen, 
sodann 24 Stunden mit einer 1\% $1 \%$ igen Natronlauge behandelt, der Pepsinsalzsäure- und der Pankreasverdauung unterworfen und nach dem Auswaschen mit Wasser, mit Alkohol und Aether von seinem Farbstoff befreit worden.

Es gab die Biuretreaction an der festen Substanz zwar nicht sehr intensiv, aber positiv, es färbte sich mit Millon's Reagens beim Erwärmen roth und gab die Xanthoproteïnreaction. Mit concentrirter Salzsäure färbte es sich nicht violett, ebenso nicht mit Eisessig und concentrirter Schwefelsäure. Mit Kali geschmolzen, entwickelte es starken Skatolgeruch.

Da es die Biuretreaction gab, so sind wir nach den Arbeiten Kossel's berechtigt, einen Hexonkern in seinem Moleküle anzunehmen. Ich habe schon früher die relative Grösse dieses Kernes im Vergleich zu den Eiweisskörpern der Seide und zur Gelatine bestimmt. Entsprechende Bestimmungen wurden bald darauf von Hausmann ${ }^{1}$ ) für mehrere Eiweissstoffe publicirt. Hausmann bestimmte ausser dem in Form von basischen Stoffen vorhandenen Stickstoff noch den Stickstoff der Amidosäuren und den als Ammoniak abspaltbaren Stickstoff und gab damit ein vollständiges Bild für die Vertheilung des Stickstoffs im Moleküle der von ihm untersuchten Körper.

Hausmann's Verfahren für die Bestimmung des Stickstoffs im Phosphorwolframsäure-Niederschlage unterscheidet sich dadurch von meinem, dass er den Niederschlag in Alkali gelöst und mit abgemessenen Theilen dieser Lösung die Bestimmung nach Kjeldahl vorgenommen hat, während ich den Niederschlag nebst Filter im Kjeldahlkolben zerstört habe. Hausmann gibt auch Zahlen für die Gelatine an, für welche ich ebenfalls, um einen Vergleich mit den Bestimmungen Anderer zu ermöglichen, den Basenstickstoff bestimmt habe. Unsere Zahlen differiren sehr bedeutend. Ich habe 18,85\% des Gesammtstickstoffs gegenüber $35,85 \%$ bei Hausmann angegeben. Diese Differenz erklärt sich indess auf sehr ein-

1) Diese Zeitschrift, Bd. XXVII, S. 9อ̃. 
fache Weise. Die Menge Stickstoff, die man erhält, wird bei verdünnten Lösungen zu niedrig, bei sehr concentrirten $\mathrm{zu}$ hoch ausfallen, da man in dem einen Falle nicht alle Basen ausfällt, im andern ausser den Basen wahrscheinlich auch noch Amidosäuren mit ausfallen können. Diese lassen sich freilich, wenn auch schwierig, aus dem Niederschlage durch Auswaschen entfernen. Absolut genommen sind daher meine Zahlen, wie schon aus einer Bemerkung in meiner früheren Arbeit hervorgeht ${ }^{1}$ ), zu niedrig, während Hausmann's um die obere Grenze sich bewegen und annähernd den absoluten Werthen entsprechen mögen. Wenn ich unter diesen Voraussetzungen die von mir angegebene Zahl von 8,66\% Basenstickstoff für das Pinnaconchiolin in Vergleich mit den von Hausmann für Casein, Serumglobulin etc. angegebenen Zahlen setzen will, so muss ich für eine Abschätzung die Zahlen beider für die Gelatine heranziehen. Danach ergibt sich, dass das Conchiolin wahrscheinlich etwas mehr Diaminostickstoff enthält als das Casein und weniger als das Eieralbumin.

$\mathrm{Zu}$ dieser Bestimmung ist nicht das mit Alkali und durch Verdauung gereinigte, sondern das nach der Entkalkung und dem Auswaschen nur mit Alkohol und Aether behandelte rothe Pinnaconchiolin verwendet worden.

Ich habe schon vor meiner früheren Publication eine Bestimmung des ais Ammoniak abspaltbaren Stickstoffs gemacht, welche ich damals nicht mit angegeben habe, da ich sie noch auf andere Substanzen ausdehnen wollte. Meine Bestimmungen lassen auf diesem Gebiete einen direkten Vergleich mit denen Hausmann's zu, wie aus den für die Gelatine gefundenen Werthen hervorgeht. Nach Hausmann enthält Gelatine $1,61 \%$ Amidstickstoff, nach meinen Bestimmungen 1,76\%, Werthe, welche kaum ausserhalb der möglichen Analysenfehler liegen.

$\mathrm{Da}$ ich voraussichtlich keine Gelegenheit haben werde, meine Erfahrungen auf diesem Gebiete zu erweitern; so stelle

1) Diese Zeitschrift, Bd. XXVI, S. 539. 
ich hier neben die für das Conchiolin gefundenen Werthe auch die für das Fibroin und den Seidenleim gefundenen, da ich diese Zahlen für sich nicht publiciren kann. In dieselbe Tabelle bringe ich einige von Hausmann (H.) untersuchte Körper.

$\begin{array}{lcc}\text { Fibroin aus weisser Seide } & 0,42 & - \\ \text { Fibroin aus gelber Seide } & 0,56 & - \\ \text { Leim (Gelatine) } & 1,76(1,61 \mathrm{H} .) & 18,85 \text { (35,83 H.) } \\ \text { Rothes Pinnaconchiolin } & 3,47 & 8,66 \\ \text { Seidenleim aus gelber Seide } & 8,24 & 10,00 \\ \text { Eieralbumin } & 8,53 \text { (H.) } & 21,33 \text { (H.) } \\ \text { Casein } & 13,37 \text { (H.) } & 11,71 \text { (H.) }\end{array}$

Der Seidenleim enthält hiernach etwa ebenso viel Amidstickstoff, als das Eieralbumin, das Fibroin enthält sehr viel weniger, jedoch mehr als die Gelatine, das Conchiolin steht in der Mitte zwischen dem Fibroin und dem Eieralbumin. ${ }^{1}$ )

Die Analysen der Substanz ergaben folgende Werthe. Diese beziehen sich auf den bei $125-130^{\circ}$ getrockneten und aschefrei berechneten Stoff.

$0,1568 \mathrm{~g}$ Substanz gaben $0,0922 \mathrm{~g} \mathrm{H}_{2} \mathrm{O}$ und $0,3039 \mathrm{~g} \mathrm{CO}_{2}$, entsprechend $52,87 \% \mathrm{C}$ und $6,54 \% \mathrm{H}$.

0,1541 g Substanz entsprachen bei der Stickstoffbestimmung nach Kjelda hl 8,01 ccm. einer Viertelnormallauge, entsprechend $16,69^{\circ} / 0 \mathrm{~N}$.

$1 \mathrm{ccm}$. der Lauge entspricht $3,2115 \mathrm{mg} \mathrm{N}$.

0,1770 g Substanz entsprechen $8,95 \mathrm{ccm}$. derselben Lauge, entsprechend $16,51 \% \mathrm{~N}$.

$0,6355 \mathrm{~g}$ Substanz gaben $0,0392 \mathrm{~g} \mathrm{BaSO}_{4}$, entsprechend $0,85 \% \mathrm{~S}$. geführt.

Die Schwefelbestimmung wurde nach v. Asbóth ${ }^{2}$ ) aus-

\begin{tabular}{|c|c|c|c|c|}
\hline Conchiolin & $\% \mathrm{C}$ & $\% \mathrm{H}$ & $\% \mathrm{~N}$ & $\% \mathrm{~S}$ \\
\hline aus rothen Pinnaschaalen & 52,87 & 6,54 & 16,6 & $0,8 \tilde{z}$ \\
\hline $\begin{array}{l}\text { nach Enge }{ }^{3} \text { ) } \\
\text { Mittel aus seinen Analysen }\end{array}$ & $\check{5} 2,9$ & 7,55 & 16,09 & 0,472 \\
\hline
\end{tabular}

1) Beziehungen $\mathrm{zu}$ anderen Eiweisskörpern lassen sich mit Hülfe der inzwischen publicierten Tabellen von Kossel und Kutscher feststellen. Sitzungsbr. d. Gesellschaft z. Bfrdg. d. ges. Naturw. z. Marburg, vom 6. April 1900.

2) Chemikerzeitung 1895 .

3) Zeitschrift f. Biologie, Bd. 27, S. 383 u. 384. 
Die Analyse ergibt einen höheren Kohlenstoffgehalt als diejenigen Schlossberger' ${ }^{1}$ ) und Krukenberg's. ${ }^{2}$ ) Am besten stimmt sie noch zu der darunter gesetzten von Engel, welche sich aut die Grundsubstanz der Eischaalen von Murex bezieht. Auffallend ist in meiner Analyse das Vorhandensein von Schwefel, welches dem Conchiolin fehlen soll.

Die von Engel untersuchten Häute aus Perlmuscheln, welche keinen Schwefel mehr enthielten, ${ }^{3}$ ) waren zuerst mit verdünnter Salzsäure und Pepsin, dann während einer halben Stunde mit einer ${ }^{1 / 2}$ procentigen heissen Lauge, dann mit heisser Essigsäure und mit Wasser behandelt worden. Ich glaube, dass die von mir vorgenommene Behandlung der Eischaalen ebenfalls hätte genügen müssen, um Eiweisssubstanzen zu entfernen. Da trotzdem der Rest noch schwefelhaltig war, so bleibt wohl nichts übrig als die Annahme, dass der Grundstoff dieser Schaalen in der That ein schwefelhaltiges Albuminoïd ist oder wenigstens, ähnlich wie die Schaalen von Murex nach Eng el, neben einem schwefelfreien noch einen schwefelhaltigen enthält.

Ich habe das so gereinigte Conchiolin noch einmal mit einer Zehntelnormalnatronlauge eine Woche lang extrahirt. Auch der jetzt erhaltene ungelöste Stoff gab bei der Schönn'schen Probe noch Violettfärbung mit Nitroprussidnatrium und Braunfärbung beim Zusatz alkalischer Bleilösung. Er enthielt also ebenfalls noch Schwefel. Eine Bestimmung der Menge dieses Schwefels ist nicht gemacht worden. - Dass die gefundenen $0,85 \% \mathrm{~S}$ nicht auf dem geringen Aschegehalt von $0,8 \%$ beruhen können, geht schon daraus hervor, dass selbst durch die Annahme, dass die ganze Asche aus schwefelsaurem Salz bestünde, nur ein kleiner Bruchtheil des Schwefels seine Erklärung fände.

Indessen lassen sich die widersprechenden Angaben durch die Annahme erklären, dass der Schwefel des Conchiolins nicht als Bleischwefel gewonnen werden kann, sondern in

1) Chemie der Gewebe. S. 234.

2) B. d. d. ch. G. Bd. XVIII.

3) Zeitschrift f. Biologie, Bd. 28, S. 351. 
einer andern Form gebunden ist. Ueber die verschiedenen Bindungsweisen des Schwefels im Eiweiss sind wir besonders durch die Arbeiten von $\mathrm{Krüger}$ und von Schulz aufgeklärt worden. Auf Grund dieser Arbeiten erklärt es sich, dass Engel den im Conchiolin der Perlmuschel schwer abspaltbaren Schwefel übersehen haben kann, da er den Nachweis dadurch versucht hat, dass er das in Alkali gelöste Conchiolin mit einer alkalischen Bleilösung versetzte. Dabei trat keine Schwärzung auf. In der That gibt das von mir zur Elementaranalyse verwendete Pinnaconchiolin keine Schwärzung beim Kochen mit einer alkalischen Bleilösung.

Wenn ich auch für die Richtigkeit dieser Beobachtungen einstehe, so kann ich mich doch schwer entschliessen, definitiv die Zugehörigkeit des Schwefels zum Molekül des Conchiolins anzunehmen, da ich immer noch besorge, dass ich irgend einen Punkt nicht berücksichtigt haben könnte, aus dem die Anwesenheit des Schwefels sich durch einen nicht zum Conchiolin gehörigen Stoff erklären würde.

Anhangsweise möchte ich noch einige Angaben über die ausser dem Conchiolin in den Schaalen enthaltenen Substanzen machen.

Bei der Entkalkung mit Salzsäure tritt ein ziemlich starkes Schäumen der Entkalkungsflüssigkeit auf, welches vermuthlich auf der Anwesenheit gelöster organischer Produkte beruht. Diesem Körper bin ich nicht weiter nachgegangen.

Die entkalkte Grundsubstanz enthält bei Mytilus einen schwarzen, bei Pinna einen rothen Farbstoff. Beide sind völlig verschieden von einander.

\section{Der Farbstoff aus den rothen Pinnaschaalen.}

Aus den zerkleinerten, nicht entkalkten Schaalen lässt sich mit Alkohol kein Farbstoff extrahiren. Auch wenn der Alkohol mit Salzsäure versetzt ist, ändert dies nichts.

Aus den mit Säure entkalkten Schaalen dagegen lässt sich der gelbrothe Farbstoff mit Alkohol sowohl wie mit Aether 
extrahiren. Die Extraction gelang nie vollständig, auch nach stundenlanger Extraction im Soxleth'schen Extractionsapparat blieben die Schaalen noch röthlich gefärbt. Ausser in Aether und Alkohol erwies sich der Stoff noch als in Chloroform und in Olivenöl löslich. In Wasser ist er unlöslich. Giesst man die alkoholische Lösung in viel Wasser, so scheidet er sich in Flocken aus und lässt sich abfiltriren.

Die alkoholische Lösung gibt eine diffuse Absorption im blauen Theile des Spectrums, jedoch treten keine $\mathrm{Ab}$ sorptionsbänder auf.

Seiner Löslichkeitsverhältnisse wegen wird der Farbstoff den Lipochromen anzureihen sein.

\section{Der schwarze Farbstoff der Mytilusschaalen.}

Beim Kochen der Schaalen mit Säuren bleibt eine schwarze Masse ungelöst. Ein Theil des schwarzen Körpers geht in die Lösung über. Dieses liess sich aus einer Probe der Flüssigkeit durch Zusatz von Kupfersulfat ausfällen und setzte sich in Flocken ab. Die darüber stehende Flüssigkeit bleibt dann noch schwach gelb gefärbt. Der in Lösung gegangene Farbstoff ist nicht gewonnen worden, meine Angaben beziehen sich nur auf das ungelöste schwarze Pigment.

Ich muss es unentschieden lassen, ob dieser schwarze Stoff im Wesentlichen dem ursprünglichen Farbstoff entspricht oder als neugebildete Melaninsäure anzusehen ist. Da letztere nach Schmiedeberg stets nur in geringer Menge gebildet wird, so ist es nicht ausgeschlossen, dass wir es im Wesentlichen mit dem ursprünglichen Farbstoff zu thun haben. Ich habe mich darauf beschränkt, ein reineres Produkt aus dem Bodensatze zu gewinnen und dessen Eigenschaften festzustellen.

Mikroskopisch zeigten die gröberen Theile des Bodensatzes eine faserige Structur, es waren also noch unzersetzt gebliebene Antheile des äusseren Blattes der Mytilusschaalen. Diese werden also durch 24stündiges Kochen mit Schwefelsäure nicht völlig in Lösung gebracht. Der abfiltrirte und schwefel- 
säurefrei gewaschene Niederschlag gab folgende analytische Resultate:

$$
57,5^{\circ} \mathrm{C}, 5,7 \% \mathrm{H}, 10,6 \% \mathrm{~N} \text {. }
$$

Der hohe Kohlenstoffgehalt deutet den Charakter eines Melanins an.

Indes besteht die Substanz noch aus zwei verschiedenen Stoffen. Dies geht daraus hervor, dass die Theilchen derselben nach mehrmaliger Extraction mit Natronlauge quellen und dann eine hellbraun durchscheinende Masse darstellen, also nicht wie das Melanin rein schwarz sind. Ferner zeigt die Analyse der durch Auflösen in Natronlauge und Wiederausfällen mit Säuren gereinigten Substanz eine erheblich andere Zusammensetzung.

Die reine Substanz habe ich auf folgendem Wege gewonnen. Das abfiltrirte und ausgewaschene rohe Melanin wurde mit einer Viertelnormallauge übergossen und verrieben, darauf centrifugirt. Ein bedeutender Theil war in Lösung gegangen. Von dem Bodensatz wurde vorsichtig abgegossen und die tiefschwarze Flüssigkeit noch mehrmals filtrirt. Eine Probe der Flüssigkeit gab, mit viel Wasser bis zur Durchsichtigkeit verdünnt, eine weinrothe völlig klare Lösung, aus der sich auch bei längerem Stehen nichts absetzte.

Aus dieser Lösung lässt sich der Stoff mit Schwefelsäure, Salpetersäure, Salzsäure und Essigsäure fällen. Es ist daher eine schwache Säure und er darf daher kurz als Melaninsäure bezeichnet werden. Aus der genau neutralisirten Flüssigkeit wird die Melaninsäure mit Lösungen von Kupfersulfat, Silbernitrat und Quecksilberchlorid in Form der betreffenden Metallsalze ausgefällt. Von diesen habe ich das Kupfersalz in Mengen von einigen Decigrammen gewonnen. Es löste sich in verdünnter Natronlange und wurde mit Schwefelwasserstoff zersetzi. Nach dem Abfiltrieren des Schwefelkupfers erwies sich der Aschenrückstand des eingedampften und veraschten Filtrates als kupferfrei. Dies Verfahren dürfte daher zur Umfällung und Reinigung der Melaninsäure anwendbar sein.

Die durch Auflösen in Natronlauge, Abcentrifugiren des unlöslichen Theils und Ausfällen mit Schwefelsäure gewonnene 
Melaninsäure zeigte in verschiedenen Präparaten, welche durch gesonderte mehrmalige Extraction des schwarzen Gesammtrückstandes mit Natronlauge gewonnen wurden, eine ziemlich constante Acidität. Diese wurde bestimmt durch Auflösen in einem Ueberschuss einer Zehntelnormalnatronlauge und Zurücktitriren mit einer Zehntelnormalschwefelsäure. Die Zahlen für die Acidität bezeichnen die Anzahl Cubikcentimeter der Lauge, welche zur Neutralisation von $100 \mathrm{~g}$ der lufttrockenen Substanz erforderlich sein würden. Die Bestimmungen sind jedesmal mit einigen Decigrammen ausgeführt worden. Als Endreaction wurde der Zeitpunkt benutzt, wo ein Tropfen der Lösung, auf Curcumapapier gebracht, dieses nicht mehr bräunte. Wegen der dunklen Farbe der Flüssigkeit erforderte die Beobachtung dieses Momentes grosse Aufmerksamkeit.

Die Bestimmungen ergaben:

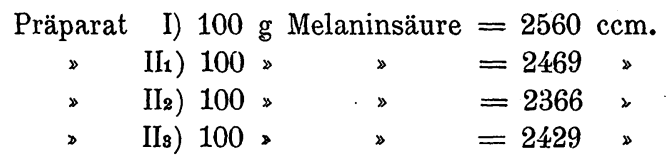

Die Analyse der bei $110^{\circ}$ getrockneten Substanz ergab: $62,05 \% \mathrm{C}, 3,9 \% \mathrm{H}, 7,7 \% \mathrm{~N}$.

Diese Werthe weichen von denen des unreinen Präparates erheblich ab und beweisen damit wiederum, dass in der ungelöst gebliebenen schwarzen Masse sich ausser der Melaninsäure noch ein anderer Stoff befindet.

Die Melaninsäure löste sich mit Leichtigkeit in einer Zehntelnormalnatronlauge, ebenso in Ammoniak und in Lösungen von kohlensaurem Natron. Die leichte und vollständige Löslichkeit in Natronlauge spricht neben der Uebereinstimmung der Aciditätswerthe für das Fehlen fremder Beimengungen.

Die analysirten Produkte enthielten noch Asche, welche nicht bestimmt worden ist.

Eisen enthält die Substanz nicht. Ich habe mehrere Decigramm, den Angaben von Röhmann und Steinitz ${ }^{1}$ ) entsprechend, mit Ammoniumnitrat und concentrirter Schwefel-

1) Zeitschrift f. analytische Chemie, Jahrg. XXXVIII, S. 433. 
säure nach Neumann ${ }^{1}$ ) verbrannt, die Reactionsmasse in Wasser gelöst, mit Ammoniak übersättigt und mit Salmiaklösung und Schwefelammonium versetzt. Es entstand keine Spur eines Niederschlages oder einer Schwärzung.

Ich weiss sehr wohl, dass die hier gegebenen Facta noch nicht zur Charakterisirung der Melaninsäure ausreichen und der Ergänzung bedürfen. Da ich aber wenig Aussicht darauf habe, diese Untersuchungen noch einmal wieder aufnehmen $\mathrm{zu}$ können, so gebe ich die angeführten Thatsachen in dem Wunsche, dass ein physiologischer Chemiker, der sich später einmal an die Untersuchung dieses Stoffes machen sollte, schon einiges zur Orientirung vorfinden möge.

\section{Zusammenfassung der Resultate.}

1. Das Conchiolin liefert als Zersetzungsprodukte mit Schwefelsäure Tyrosin, Leucin (Amidocapronsäure) und Glykokoll. Das Auftreten von Phenylamidopropionsäure oder einer andern Phenylamidosäure ist nicht wahrscheinlich. S. 396-401.

2. Es enthält einen Hexonkern (Biuretreaction und mit Phosphorwolframsäure fällbare Spaltungsprodukte). Die Menge der basischen Produkte weist ihm seine Stellung zwischen Casein und Eieralbumin an. S. 402-404.

Die Menge des in Form von Ammoniak abspaltbaren Stickstoffs beträgt 3,47\% des Gesammtstickstoffs. S. 404 .

3. Es enthält einen aromatischen Kern im Molekül (Rothfärbung mit Millon'schem Reagens, Fäkalgeruch der Kalischmelze, Amwesenheit von Tyrosin unter den Spaltungsprodukten.) S. 401/402 und 396/397.

4. Der organische Rest der Perlmuttersubstanz der Muschelschaalen unterscheidet sich von den übrigen organischen Schaalenresten durch einen geringeren Kohlenstoffgehalt. S. 392 und 393 .

1) Archiv f. Anatomie u. Physiologie, Physiolog. Abtheilg., 1897, S. 552. 